\title{
Competencias interculturales del profesorado de la enseñanza básica en Machala, Ecuador
}

Eudaldo E. Espinoza-Freire ${ }^{1}$ y Jorge L. León-González².

(1) Facultad de Ciencias Sociales, carrera de Educación Básica, Universidad Técnica de Machala. Machala, Av. 25 de Junio Km.51⁄2 vía a Pasaje, Ecuador. (Correo-e: eespinoza@utmachala.edu.ec).

(2) Editorial Universo Sur, Universidad de Cienfuegos, sede Conrado Benítez, Ave 20, Punta Gorda, Cienfuegos, Cuba. (Correo-e: jlleon@ucf.edu.cu).

Recibido Ago. 19, 2020; Aceptado Oct. 15, 2020; Versión final Nov. 9, 2020, Publicado Feb. 2021

\begin{abstract}
Resumen
El objetivo de este estudio descriptivo fue analizar las competencias interculturales de los docentes de la educación básica de la ciudad de Machala (Ecuador). Se determinaron las dimensiones cognitiva, procedimental y actitudinal y el estudio fue sistematizado mediante los métodos analítico-sintético, inductivodeductivo y estadístico. La información se obtuvo mediante una encuesta a 442 docentes seleccionados aleatoriamente a través de muestreo estratificado. Entre los resultados se destaca el dominio de las normativas y leyes que rigen la educación intercultural bilingüe, el desconocimiento de metodologías adecuadas para esta enseñanza y las limitaciones para la instrucción en las lenguas originarias. Se concluye que la mayoría de los educadores que integraron la muestra perciben tener conocimientos y sensibilidad hacia la cultura propia y la del alumnado, pero opinan poseer insuficiencias y limitaciones en cuanto a metodologías y competencias interculturales, por lo que prevalece la asimetría entre la teoría y la práctica.
\end{abstract}

Palabras clave: competencias; educación intercultural; enseñanza básica; aprendizaje; docentes

\section{Intercultural competences of primary education teachers in Machala, Ecuador}

\begin{abstract}
The objective of the present descriptive study was to analyze the intercultural competences of primary education teachers in Machala city (Ecuador). Cognitive, procedural, and attitudinal dimensions were determined and the study was systematized using analytical-synthetic, inductive-deductive, and statistical methods. Data was gathered through a survey of 442 randomly selected teachers through stratified sampling. The results showed teacher command on the regulations and laws that govern bilingual intercultural education. But, there was lack of knowledge about adequate methodologies for intercultural teaching. There were also limitations for instructing in native languages. It is concluded that the majority of surveyed teachers perceived that they had knowledge and showed sensitivity about their own cultural identity and that of their students. But, teachers also believed that they were limited in terms of intercultural methodologies and competences, which may explain the prevalent existing asymmetry between theory and practice.
\end{abstract}




\section{INTRODUCCIÓN}

A partir de la década de los años 90 del pasado siglo XX el tema de la interculturalidad ha ganado especial relevancia a nivel mundial; interés despertado por diversos factores, a saber, las actuales corrientes migratorias, el desarrollo tecnológico que ha impactado en la comunicación a nivel global y la reivindicación de los pueblos originarios. Particularmente en los países latinoamericanos la atención se centra en las relaciones de los pueblos que conviven en los espacios multiculturales; lo que se evidencia en el aumento de las actividades académicas, investigaciones científicas y en la prolífica producción científica, que abarca todas sus dimensiones (ética, política, social, religiosa, económica, epistemológica y pedagógica); así como en la implementación de políticas públicas por los estados de muchas de estas naciones suscitando polémicas y los más variados criterios.

Santoro (2014) y Rueda et al. (2019) perciben que, aunque las naciones diseñan políticas públicas encausadas al cumplimiento de los lineamientos, que en materia de interculturalidad emanan de instituciones y organizaciones internacionales como la Unesco, éstas no son contextualizadas a la realidad de cada país, lo que constituye un obstáculo para la satisfacción de las necesidades sociales, políticas, económicas y educativas, entre otras, de las poblaciones originarias. En muchas ocasiones estas políticas públicas se enfocan de manera reduccionista en la atención a las necesidades básicas de los grupos culturales excluidos socialmente; pero, es más que esto, la interculturalidad es una vía de emancipación y empoderamiento de los miembros de las comunidades socioculturales menos favorecidas para lograr una mejor inclusión y participación en la construcción de una sociedad diversa, justa y equitativa, en la cual prime el respeto a los derechos humanos (Sleeter, 2014; Tarozzi, 2014).

Una verdadera interculturalidad existe cuando se construyen nuevas relaciones sociales verdaderamente igualitarias, que tengan en cuenta las diferencias socioculturales de los pueblos que conviven en un mismo espacio, como una expresión de democracia (Garita, 2014). En tal sentido, Moreno (2018, p.4) considera que la interculturalidad es "la forma más desarrollada del diálogo de las culturas y las civilizaciones"; lo que solo será posible lograr con el empoderamiento de los grupos que históricamente fueron relegados. Criterio compartido con Boni y Calabuig (2017), y Rueda et al. (2019), quienes además sostienen que la educación intercultural debe estar unida a estos procesos emancipadores para que ofrezca posibilidades de empoderamiento a los grupos relegados y así lograr su participación inclusiva como ciudadanos en la construcción social, diversa, justa, equitativa y de respeto de los derechos humanos. Razón por la cual Boni et al. (2016) estiman que se debe educar interculturalmente desde las aulas para que el alumnado aprenda a respetar la diversidad cultural y pueda alcanzar las competencias interculturales necesarias para vivir en paz en un ambiente multicultural.

Ahora bien, ¿qué se entiende por competencias interculturales? La respuesta a esta pregunta la podemos encontrar siguiendo a Levy-Leboyer (1996), quien las define como las capacidades del individuo para saber ser y hacer en un contexto multicultural; son los conocimientos, habilidades y actitudes que le permiten llevar a cabo las actividades y funciones personales, profesionales y afectivas para establecer relaciones con diferentes culturas. Estas competencias necesitan ser formadas desde una enseñanza intercultural; para Aguado (1991) educar interculturalmente es lograr la inclusión de todos los ciudadanos erradicando las formas de poder fundamentadas en la diferencia entre culturas y construyendo espacios de convivencia caracterizados por la igualdad de condiciones y oportunidades para todos. El reconocimiento de los derechos de la cultura del otro, el respeto a su cosmovisión, son elementos cruciales en la educación intercultural. Su principal objetivo es facilitar a cada ciudadano la construcción de su identidad y aprender a apreciar y respetar la de los demás, lo que significa reconocer sus valores, saberes, tradiciones e historia.

Sobre el tema de las competencias interculturales en el ámbito pedagógico se han realizado múltiples investigaciones; entre ellas las llevadas a cabo por Schweisfurth y Gu (2009), Boni et al. (2016), Krainer et al. (2017) y Rueda et al. (2019), quienes desde diversas metodologías y enfoques enfatizan en la necesaria orientación del proceso de enseñanza-aprendizaje hacia las dimensiones cognitiva, procedimental y actitudinal, en un contexto de intercambio de las diversas culturas que conviven en el aula, en busca de competencias interculturales, las que confluyen y se manifiestan a través de la conducta del estudiante. En tal sentido, Aguado (1991) considera que es necesario contar con docentes que dominen las particularidades de cada cultura, pero sobre todo que sean portadores de valores éticos, en los cuales no tengan espacio la discriminación y la exclusión por razones de origen.

Criterio que se corresponde con los resultados de los estudios de Uribe-Pérez y Mosquera (2016), y Espinoza (2020), en los que se evidencian que las actitudes de los maestros y profesores hacia la diversidad cultural están en correlación con la formación recibida; por lo que se hace imprescindible que los programas de estudio para las carreras pedagógicas contemplen entre sus contenidos conocimientos de temas tales como culturas originarias, normativas de la educación intercultural y políticas públicas interculturales. En relación con la dimensión procedimental deben ser desarrolladas habilidades para: propiciar ambientes áulicos 
interculturales caracterizados por el intercambio de saberes y el respeto a lo diverso, diseñar e implementar estrategias educativas que faciliten la integración de los conocimientos desde la cosmovisión de los alumnos procedentes de diferentes culturas, realizar investigaciones relacionadas con la interculturalidad e impartir las clases en la misma lengua de los alumnos, entre otras. En el plano axiológico o dimensión actitudinal se han de fomentar principios interculturales y valores éticos y cívicos como contribución a la efectividad de la educación intercultural.

En el caso del Ecuador, su condición de país multicultural y plurinacional se consagra en la Carta Magna del 2008, al estipular que "El Ecuador es un Estado constitucional de derechos y justicia, social, democrático, soberano, independiente, unitario, intercultural, plurinacional y laico..." (Asamblea Nacional Constituyente de la República del Ecuador, 2011, art. 1); este postulado se sistematiza a través de códigos y leyes que rigen la vida del país. En la esfera educativa, el Ministerio de Educación promulgó la Ley Orgánica de Educación Intercultural (LOEI) en el 2011, como instrumento normativo y político que potencia el sistema de educación bilingüe en todos los niveles de enseñanza, facilitando espacios educativos de diálogo entre las diversas culturas que integran la sociedad ecuatoriana, para así garantizar la preservación de las identidades de los pueblos y naciones que comparten el espacio multicultural del país (Asamblea Nacional de la República del Ecuador, 2016).

Sin embargo, no es suficiente contar con un apropiado marco legal para que exista una plena educación intercultural en la cual fundamentar el desarrollo de competencias interculturales; se perciben falencias en la práctica escolar, basta mencionar las limitaciones y desidia en el tratamiento de lo indígena, subvaloración de las culturas ancestrales, escaso empleo de técnicas y estrategias de enseñanza-aprendizaje interculturales, poco empleo de saberes ancestrales y experiencias que permitan enriquecer los conocimientos y desarrollar las habilidades interculturales, no empleo de las lenguas originarias en el proceso de enseñanza e irrespeto de las culturas indígenas a través de manifestaciones de bullying hacia los estudiantes procedentes de pueblos originarios (Espinoza et al., 2019).

Investigadores como Aman (2017) y Krainer et al. (2017) destacan la necesidad de superar estas dificultades en el orden objetivo; pero, sobre todo se precisa de un cambio de mentalidad de los actores sociales, lo que requiere de una educación en valores humanos, donde el respeto sea un pilar fundamental. La construcción de una nueva sociedad, donde se tenga en cuenta la sabiduría de todos los pueblos y naciones que la habitan, constituye un reto, no sólo en el orden epistemológico, sino y con mayor énfasis en el práctico. Si bien el proceso de cambio requiere de la transformación de estructuras y voluntad política, es indiscutible que sin el desarrollo de competencias interculturales y una profunda convicción de la oportunidad que representa la integración de culturas para el desarrollo del país en todos los órdenes, el verdadero diálogo intercultural no se podrá concretar en la práctica (Krainer et al. 2017).

Para alcanzar estas competencias interculturales la escuela es un espacio en el que se pueden introducir estrategias de inclusión partiendo de la diversidad cultural de los estudiantes. Según Herrera et al. (2016), la escuela es un espacio donde concurren formas diversas de culturas, por lo que la educación intercultural es una alternativa que debe ser empleada en la socialización y transformación cultural. Desde esta perspectiva, la educación intercultural es una posible respuesta a la convivencia multicultural. En este sentido, las instituciones educativas juegan un rol protagónico, pues en sus espacios se forman las nuevas generaciones de ecuatorianos y ecuatorianas, sin olvidar que la institución educativa es un actor de influencia de su entorno social.

Ahora bien, para poder lograr este rol de la escuela, se necesita de los conocimientos, capacidades y actitudes de los docentes en torno al enfoque de la educación intercultural; así como del reconocimiento de los atributos de las culturas diversas que representan los estudiantes; de ahí la pertinencia e importancia de este estudio cuyo objetivo es analizar las competencias interculturales de los docentes de la Enseñanza Básica de la ciudad de Machala, como parte de una investigación que se lleva a cabo por un grupo de profesores de la carrera de Educación Básica de la Universidad Técnica de Machala (UTMach), para sobre el análisis de los resultados del diagnóstico implementar acciones, que contribuyan al perfeccionamiento del currículo de la formación inicial y a la capacitación postgraduada de los docentes, para así contribuir al logro de competencias interculturales y un verdadero diálogo intercultural desde la actividad pedagógica.

\section{METODOLOGÍA}

La estrategia investigativa asumida responde a un estudio descriptivo con enfoque cuantitativo sustentado en los métodos de investigación científica analítico-sintético y estadístico. El análisis y síntesis permitió estudiar los referentes teóricos que fundamentan el estudio, los que fueron resumidos para la construcción del discurso escrito, la discusión de los resultados y arribar a las conclusiones. El método estadístico sirvió para planificar, recolectar, procesar y analizar los datos obtenidos mediante una encuesta aplicada a un grupo de docentes seleccionados de las escuelas de la Enseñanza Básica, de la ciudad de Machala. 
Esta estrategia investigativa se respaldó en la metodología de investigación científica de Hernández-Sampieri et al. (2014), quienes declaran que el propósito de las investigaciones descriptivas es narrar cómo son y se manifiestan las situaciones, eventos, fenómenos, personas, grupos o comunidades. "Miden o evalúan diversos aspectos, dimensiones o componentes del fenómeno o fenómenos a investigar" (HernándezSampieri et al., 2014, p.66).

El problema de investigación se centra en la ausencia de conocimientos sobre el nivel de competencias interculturales de los docentes de la Enseñanza Básica, de la ciudad de Machala, formulándose la siguiente pregunta de investigación ¿Cuál es el nivel de competencias interculturales de los docentes de la Enseñanza Básica, de la ciudad de Machala? y como hipótesis descriptiva que el nivel de competencias interculturales de los docentes de la Enseñanza Básica, de la ciudad de Machala se caracteriza por los conocimientos y actitudes para la educación intercultural, no así desde la dimensión procedimental.

La lógica de las averiguaciones realizadas busca dar respuestas a las siguientes preguntas:1) ¿Qué criterios se manejan en la bibliografía especializada sobre el desarrollo de las competencias interculturales? 2) ¿Cuál es el nivel actual de los conocimientos interculturales de los docentes de la Enseñanza Básica, de la ciudad de Machala? 3) ¿Cómo se comporta la dimensión procedimental de las competencias interculturales en los docentes de la Enseñanza Básica, de la ciudad de Machala? 4) ¿Cuál es nivel actual de la dimensión actitudinal de las competencias interculturales en los docentes de la Enseñanza Básica, de la ciudad de Machala?

\section{Instrumento de recogida de información}

La elaboración de la encuesta se realizó siguiendo el esquema propuesto por Hernández-Sampieri et al. (2014), la cual consta de las siguientes etapas: 1) revisión en la literatura especializada sobre instrumentos afines a la investigación; 2) evaluar la validez de estos instrumentos y posibilidades de contextualización; 3) indicar los niveles de medición de preguntas y escalas; 4) determinar la codificación de preguntas cerradas; 5) elaborar la primera versión de los instrumentos; 6) consultar con expertos o personas familiarizadas con el tema de investigación; 7) ajustar la primera versión; 8) entrenar a los miembros del equipo que aplicarán los instrumentos; 9) prueba piloto; y 10) elaborar la versión final.

La encuesta tuvo como objetivo obtener información sobre los criterios de los profesores respecto a sus competencias interculturales; contó con 34 preguntas, agrupadas según las dimensiones: cognitiva (perspectiva de interculturalidad que posee el docente y la de sus alumnos), procedimental (estrategias educativas, métodos y procedimientos culturalmente apropiados que aplica el profesor) y actitudinal (conciencia que tiene el educador de sus propios valores y referentes culturales: así como la percepción que de estos tienen sus alumnos). Las respuestas a las preguntas fueron seleccionadas mediante una escala likert, de 0 (Muy bajo) a 10 (Muy alto).

Este instrumento contó para su elaboración con los referentes metodológicos y conceptuales de los instrumentos: cuestionario para futuros docentes de Educación Secundaria acerca de las percepciones sobre atención a la diversidad, validado por Colmenero y Pegalajar (2015); cuestionario sobre las competencias interculturales de profesores virtuales propuesto por Ricardo et al. (2017) y encuesta aplicada por Rueda et al. (2019) en el estudio intitulado "Análisis de la Educación Intercultural en grupos de estudiantes de la Universidad Francisco de Paula Santander, en Colombia, que fueron víctimas del conflicto armado"; las aportaciones de estos sirvieron de referentes para la elaboración del instrumento contextualizado a la Enseñanza Básica de la ciudad de Machala.

Una vez diseñada la primera versión del cuestionario se sometió al criterio de expertos para su validación (Método Delphi). El grupo de expertos estuvo integrado por siete especialistas en el tema, cuatro pedagogos, dos sociólogos y un psicólogo, de ellos cuatro doctores en ciencia y el resto master; asimismo, todos cuentan con categorías docentes principales (titulares o auxiliares). En la primera ronda los expertos evaluaron el instrumento siguiendo los siguientes parámetros: a) materialización de los referentes teóricos de las competencias interculturales a través de las preguntas; b) alcance de las peguntas del instrumento como sistema; c) concepción estructural y metodológica del cuestionario que permite su correcta implementación en la práctica; d) requerimientos técnicos y humanos para aplicar el instrumento y e) aceptación del instrumento como vía para la recepción de la información.

Estos aspectos se evaluaron con las categorías de: muy adecuado (MA), bastante adecuado (BA), adecuado (A), poco adecuado (PA) e inadecuado (I); de esta forma fueron evaluados de A los aspectos a, b, c y e; de $\mathrm{BA}$ el d. Además, se recogieron los criterios, sugerencias, puntos de vista y argumentos sobre los elementos que a consideración de los expertos debían ser mejorados o modificados. Entre estas sugerencias se significaron la inclusión de preguntas sobre el dominio de conocimientos metodológicos para lograr los objetivos de la educación intercultural bilingüe por parte de los docentes y la división por dimensiones de las 
preguntas del cuestionario. Una vez hecha las correcciones pertinentes se sometió a los expertos la segunda versión, los que evaluaron los aspectos b y e de BA y los a, c y d como MA. Los expertos concluyeron que su aplicación era pertinente para la recogida de información necesaria para diagnosticar el nivel de las competencias interculturales de los docentes. También, se determinó la confiabilidad del instrumento mediante la prueba del coeficiente Alfa de Cronbach, obteniendo los siguientes valores por dimensión:

Tabla 1: Confiabilidad de la encuesta

\begin{tabular}{|l|c|}
\hline \multicolumn{1}{|c|}{ Dimensión } & Valor de Alfa de Cronbach \\
\hline Cognitva & 0.953 \\
\hline Procedimental & 0.952 \\
\hline Actitudinal & 0.972 \\
\hline
\end{tabular}

Una vez entrenados los integrantes del equipo de investigación y hecha la prueba piloto se aplicó la encuesta en los colegios donde prestan servicios los docentes seleccionados en un horario previamente coordinado con los directivos de estos centros. Los datos obtenidos se procesaron con el auxilio del programa estadístico SPSS versión 21, los que fueron resumidos mediante frecuencias absolutas y relativas, y presentados en tablas descriptivas.

\section{Población y muestra}

La población del estudio estuvo constituida por los 1732 docentes de la Enseñanza Básica de la ciudad de Machala. El tamaño de la muestra fue calculado mediante la fórmula: $n=N Z^{2} p q /\left(e^{2}(N-1)+Z^{2} p q\right)$; donde: $n=$ tamaño de la muestra; $N=$ tamaño de la población $(\mathrm{N}=1732) ; \mathrm{Z}=$ constante correspondiente al nivel de confianza $(Z=1.96$ para un nivel de confianza del $95 \%)$; $e=e r r o r$ muestral $(e=0.04) ; p=$ probabilidad de éxito $(p=0.95) ; y q=$ probabilidad de fracaso $(q=0.05)$; resultado un tamaño de 445 unidades de observación. Para garantizar la representatividad de todos los tipos de escuelas se aplicó el muestro estratificado según metodología de Hernández-Sampieri et al. (2014). Se consideraron como estratos cada uno de los tipos de colegios (fiscales, particulares y fiscomisionales). Después de aplicado el instrumento, se descartaron 3 encuestas por presentar errores en su llenado o información incompleta, de esta forma la muestra quedó finalmente conformada por 442 docentes tal como se puede observar en la tabla 2.

Tabla 2: Muestra de los docentes de la Enseñanza Básica.

Ciudad de Machala. Período Académico 2018-2019

\begin{tabular}{|l|c|c|}
\hline \multicolumn{1}{|c|}{ Tipo de escuela } & Cantidad de profesores & Profesores seleccionados \\
\hline Fiscales & 1105 & 282 \\
\hline Particulares & 415 & 106 \\
\hline Fiscomisionales & 212 & 54 \\
\hline Total & 1732 & 442 \\
\hline
\end{tabular}

\section{RESULTADOS Y DISCUSIÓN}

El análisis y la discusión de los resultados se realizan a continuación atendiendo a las dimensiones de las competencias interculturales de los docentes para el ejercicio de la educación intercultural (cognitiva, procedimental y actitudinal). Los datos obtenidos a través de la encuesta fueron sintetizados en cinco categorías Muy Bajo (0-3), Bajo (4 ○ 5), Medio (6 o 7), Alto (8 ○ 9) y Muy Alto (10), tal como se muestra en las siguientes tablas descriptivas.

\section{Dimensión cognitiva}

A continuación se resumen en la tabla 3 los criterios de los docentes seleccionados, en los centros educativos de la Enseñanza Básica de la ciudad de Machala, sobre el conocimiento de los aspectos relativos a la dimensión cognitiva de las competencias interculturales necesarias para el desarrollo de la educación intercultural. 
Tabla 3: Resultados de la dimensión cognitiva.

\begin{tabular}{|c|c|c|c|c|c|c|c|c|c|c|}
\hline \multirow{3}{*}{ Indicador } & \multicolumn{10}{|c|}{ Escala } \\
\hline & \multicolumn{2}{|c|}{ Muy Bajo } & \multicolumn{2}{|c|}{ Bajo } & \multicolumn{2}{|c|}{ Medio } & \multicolumn{2}{|c|}{ Alto } & \multicolumn{2}{|c|}{ Muy Alto } \\
\hline & Cant. & $\%$ & Cant. & $\%$ & Cant. & $\%$ & Cant. & $\%$ & Cant. & $\%$ \\
\hline $\begin{array}{l}\text { Conocimiento sobre las normativas } \\
\text { constitucionales sobre interculturalidad. }\end{array}$ & 9 & 2,0 & 24 & 5,4 & 255 & 57,7 & 145 & 32,8 & 9 & 2,0 \\
\hline Conocimiento sobre la LOEI. & 5 & 1,1 & 10 & 2,3 & 258 & 58,4 & 163 & 36,9 & 6 & 1,4 \\
\hline $\begin{array}{l}\text { Conocimiento sobre las políticas } \\
\text { educativas implementadas en la escuela } \\
\text { y su eficacia en la promoción de equidad } \\
\text { de condiciones en el proceso educativo. }\end{array}$ & 14 & 3,2 & 21 & 4,8 & 255 & 57,7 & 133 & 30,1 & 19 & 4,3 \\
\hline $\begin{array}{l}\text { Conocimiento de sus alumnos sobre la } \\
\text { educación intercultural. }\end{array}$ & 87 & 19,7 & 144 & 32,6 & 196 & 44,3 & 8 & 1,8 & 7 & 1,6 \\
\hline $\begin{array}{l}\text { Perspectiva del aula como espacio de } \\
\text { diálogo sin restricciones por condición } \\
\text { socioeconómica o cultural del alumnado. }\end{array}$ & 43 & 9,7 & 32 & 7,2 & 232 & 52,5 & 118 & 26,7 & 17 & 3,8 \\
\hline $\begin{array}{l}\text { Conocimientos metodológicos para logar } \\
\text { los objetivos de la educación intercultural } \\
\text { bilingüe. }\end{array}$ & 211 & 47,7 & 121 & 27,4 & 109 & 24,7 & 1 & 0,2 & 0 & 0,0 \\
\hline $\begin{array}{l}\text { Reconozco mis limitaciones sobre el } \\
\text { tema de interculturalidad y busco } \\
\text { capacitarme. }\end{array}$ & 23 & 5,2 & 22 & 5,0 & 201 & 45,5 & 107 & 24,2 & 89 & 20,1 \\
\hline $\begin{array}{l}\text { Conozco y comprendo la manera en que } \\
\text { la opresión, el racismo, la discriminación } \\
\text { y los estereotipos, afectan tanto a mi } \\
\text { persona como a mi práctica educativa. }\end{array}$ & 47 & 10,6 & 44 & 10,0 & 312 & 70,6 & 31 & 7,0 & 8 & 1,8 \\
\hline $\begin{array}{l}\text { Conocimiento sobre las características } \\
\text { de los grupos o comunidades culturales } \\
\text { a las que pertenecen mis alumnos } \\
\text { (experiencias y modo de vida, cultura, } \\
\text { tradiciones, etc.). }\end{array}$ & 30 & 6,8 & 201 & 45,5 & 178 & 40,3 & 21 & 4,8 & 12 & 2,7 \\
\hline $\begin{array}{l}\text { Puedo describir los principales saberes } \\
\text { tradicionales, prácticas, y la estructura } \\
\text { social de la comunidad de mis alumnos. }\end{array}$ & 97 & 21,9 & 101 & 22,9 & 101 & 22,9 & 76 & 17,2 & 67 & 15,2 \\
\hline $\begin{array}{l}\text { Conozco las influencias sociopolíticas y } \\
\text { la manera en que afectan la vida de los } \\
\text { grupos étnicos y culturales; y como } \\
\text { pueden impactar la autoestima y el } \\
\text { autopercepción de sus alumnos en los } \\
\text { procesos educativos. }\end{array}$ & 81 & 18,3 & 20 & 4,5 & 143 & 32,4 & 188 & 42,5 & 10 & 2,3 \\
\hline $\begin{array}{l}\text { Participo en talleres, cursos y } \\
\text { conferencias con la finalidad de mejorar } \\
\text { mis conocimientos y habilidades para } \\
\text { enseñar desde una perspectiva } \\
\text { intercultural. }\end{array}$ & 77 & 17,4 & 21 & 4,8 & 187 & 42,3 & 97 & 21,9 & 60 & 13,6 \\
\hline $\begin{array}{l}\text { Soy consciente de las barreras sociales e } \\
\text { institucionales que impiden el acceso a } \\
\text { oportunidades educativas de algunos } \\
\text { grupos culturales. }\end{array}$ & 12 & 2,7 & 36 & 8,1 & 238 & 53,8 & 97 & 21,9 & 59 & 13,3 \\
\hline
\end{tabular}

Los datos de la tabla 3 develan una tendencia a un nivel entre medio y alto de conocimientos por parte de los docentes del marco legal en el cual se ampara la educación intercultural, se destaca en este sentido el dominio de la LOEI, percibido entre estas categorías por el $95,3 \%$ de los docentes (58,4\% medio y $36,9 \%$ alto); asimismo ocurre con las normas constitucionales en materia de interculturalidad con un $90,5 \%$, en este indicador el $57,7 \%$ de los docentes estima tener conocimiento medio y un $32,8 \%$ de alto; de igual manera se comporta el conocimiento sobre las políticas educativas implementadas en la escuela y su eficacia en la promoción de equidad de condiciones en el proceso educativo percibido por el $57,7 \%$ de los docentes como medio y de alto por el $30,1 \%$. 
Estos resultados significan la importancia que conceden los docentes al marco normativo que respalda la educación intercultural en el país y en el cual fundamentan su labor docente, lo que se corresponde con la participación en talleres, cursos y conferencias con la finalidad de mejorar los conocimientos y habilidades para enseñar desde una perspectiva intercultural, en este sentido el $42,3 \%$ consideran haber tenido una participación media, el 21,9\% alta y el 13,6\% muy alta. En tal sentido el Consejo de Educación Superior (2017) se ha pronunciado a favor de la formación y capacitación docente sobre el tema de interculturalidad, destacado los contenidos curriculares relativos a los enfoques epistémicos del diálogo intercultural de saberes, la articulación de los fundamentos científicos-tecnológicos con las cosmovisiones y tradiciones ancestrales; así como potenciar metodologías y ambientes que favorezcan el intercambio intercultural, el respeto a la diversidad y la identidad étnica. También, se observa que un considerable número de docentes perciben tener un nivel entre medio y alto en los indicadores relativos al reconocimiento de las limitaciones sobre el tema de interculturalidad y el de las barreras sociales e institucionales que impiden el acceso a oportunidades educativas de algunos grupos culturales.

Este reconocimiento de las limitaciones sobre la interculturalidad y de las barreras que aún existen para la plena instrumentación de la educación intercultural es el primer paso para buscar el conocimiento que permita tomar partido para su eliminación. En tal sentido Krainer et al. (2017) estiman que el principal obstáculo para el logro de una verdadera educación intercultural que responda a las condiciones de un país multicultural y plurinacional como el Ecuador es el cambio de mentalidad de los ciudadanos, donde la escuela como agente social tiene el encargo de esa transformación; para ello es necesario erradicar la vieja concepción de la superioridad de las metodologías de enseñanza occidental, para que en realidad exista un intercambio de saberes entre las diferentes culturas que conviven en el salón de clase.

En tal sentido, Rueda et al. (2019) consideran crucial la preparación, los conocimientos y el enfoque que el docente posee sobre la diversidad cultural de sus alumnos y el entorno, pues estos elementos constituyen factores que junto al currículo y las políticas de la institución educativa definen el cómo se aborda e implementa la educación intercultural. Sin embargo, se aprecia desconocimiento por parte del $52,3 \%$ de los docentes del nivel de conocimiento de sus alumnos sobre la educación intercultural y de las características, saberes y tradiciones, experiencias, modo de vida y cultura de los pueblos originarios; pero sobre todo de las metodologías para alcanzar los objetivos de la educación intercultural, lo que fue reconocido por el $75,1 \%$, quienes estiman que poseen muy bajo $(47,7 \%)$ o bajo $(27,4 \%)$ conocimientos.

Este resultado sobre el desconocimiento de las metodologías de la educación intercultural se corresponde con los obtenidos por Aman (2017) y Espinoza (2020), quienes consideran existen asimetrías entre las normativas y principios interculturales que regulan la actividad pedagógica del país respecto a los currículos de la carrera de formación de docentes de la Enseñanza Básica, en ellos no se establecen las metodologías concretas para la formación intercultural y en consecuencia enfatizan en la urgente necesidad de continuar perfeccionando los currículos y metodologías para que viabilicen la concreción de los valores interculturales en la praxis educativa. Muchas investigaciones como las de Hajisoteriou (2012), Dervin (2015) y Rueda et al. (2019) también dan cuenta de que aún son insuficientes los estudios relativos a los procedimientos, métodos y estrategias metodológicas que faciliten la implementación del enfoque intercultural de la enseñanza.

\section{Dimensión procedimental}

Los datos contenidos en la tabla 4 recogen la percepción de los docentes encuestados sobre las acciones, habilidades y procedimientos empleados en función de la actividad pedagógica relacionada con la dimensión procedimental de las competencias interculturales. La información contenida en la tabla 4 desvelan las características de las competencias interculturales de los docentes desde la dimensión procedimental, significando la limitada capacidad para la aplicación de técnicas para instruir desde entornos multiculturales y empleo experiencias educativas que permiten enriquecer los conocimientos y desarrollar las habilidades interculturales, rubro en el cual el $58,4 \%$ percibe estas capacidades como muy bajas $(7,5 \%)$ o bajas $(50,9 \%)$ y el diseño y empleo de estrategias de enseñanza-aprendizaje interculturales, considerada entre baja y muy baja por el $56,8 \%$ de los docentes de la muestra. De igual manera el $62,3 \%$ es de la opinión que la frecuencia con que se desarrollan actividades extracurriculares relacionadas con la interculturalidad es muy baja (7,5\%) o baja $(54,8 \%)$, lo que evidencia que aún estas acciones son insuficientes.

Aunque, aproximadamente el $56,3 \%$ opinó que empleaba la clase como un espacio de diálogo intercultural, percepción que se sitúa entre un nivel medio y muy alto, y que el $55,4 \%$ consideró que realiza acciones para eliminar los obstáculos que conspiran contra la inclusión de los miembros de las culturas en desventaja sociocultural, contradictoramente la Integración de los conocimientos desde la diversidad cultural y cosmovisión de los alumnos es reconocida por el $51,4 \%$ como baja o muy baja. Asimismo, el $49,1 \%$ reconoce como baja $(45,0 \%)$ o muy baja $(4,1 \%)$ la participación en actividades que ayudan a desarrollar los conocimientos sobre la propia herencia cultural y en la búsqueda de alternativas para eliminar el racismo, los prejuicios y la discriminación; lo que se contradice con la precepción del $77,8 \%$ de los docentes sobre el 
interés por mejorar los conocimientos y habilidades para le enseñanza intercultural a través de la participación en talleres, cursos y conferencias con la finalidad de mejorar los conocimientos y habilidades para enseñar desde una perspectiva intercultural, indicador correspondiente a la dimensión cognitiva.

Tabla 4: Resultados de la dimensión procedimental.

\begin{tabular}{|c|c|c|c|c|c|c|c|c|c|c|}
\hline \multirow{3}{*}{ Indicador } & \multicolumn{10}{|c|}{ Escala } \\
\hline & \multicolumn{2}{|c|}{ Muy Bajo } & \multicolumn{2}{|c|}{ Bajo } & \multicolumn{2}{|c|}{ Medio } & \multicolumn{2}{|c|}{ Alto } & \multicolumn{2}{|c|}{ Muy Alto } \\
\hline & Cant. & $\%$ & Cant. & $\%$ & Cant. & $\%$ & Cant. & $\%$ & Cant. & $\%$ \\
\hline $\begin{array}{l}\text { Integro los conocimientos desde la } \\
\text { diversidad cultural y cosmovisión de mis } \\
\text { alumnos. }\end{array}$ & 71 & 16,1 & 156 & 35,3 & 205 & 46,4 & 9 & 2,0 & 1 & 0,2 \\
\hline $\begin{array}{l}\text { Empleo de la clase como espacio de } \\
\text { diálogo intercultural, sin restricciones por } \\
\text { condición socioeconómica o cultural del } \\
\text { alumnado. }\end{array}$ & 80 & 18,1 & 113 & 25,6 & 201 & 45,5 & 43 & 9,7 & 5 & 1,1 \\
\hline $\begin{array}{l}\text { Frecuencia con que desarrolla } \\
\text { actividades extracurriculares } \\
\text { relacionadas con la interculturalidad } \\
\text { (talleres, seminarios, eventos científicos } \\
\text { estudiantiles, foros y visitas a } \\
\text { comunidades originarias y museos } \\
\text { relacionados con la temática } \\
\text { intercultural, etc). }\end{array}$ & 33 & 7,5 & 242 & 54,8 & 151 & 34,2 & 14 & 3,2 & 2 & 0,5 \\
\hline $\begin{array}{l}\text { Participo en actividades que ayuden a } \\
\text { desarrollar mis conocimientos sobre mi } \\
\text { propia herencia cultural y que buscan } \\
\text { eliminar el racismo, los prejuicios y la } \\
\text { discriminación. }\end{array}$ & 18 & 4,1 & 199 & 45,0 & 205 & 46,4 & 16 & 3,6 & 4 & 0,9 \\
\hline $\begin{array}{l}\text { Aplico técnicas de enseñanza- } \\
\text { aprendizaje para instruir desde entornos } \\
\text { multiculturales; empleo experiencias } \\
\text { educativas que permiten enriquecer los } \\
\text { conocimientos y desarrollar las } \\
\text { habilidades interculturales. }\end{array}$ & 33 & 7,5 & 225 & 50,9 & 173 & 39,1 & 10 & 2,3 & 1 & 0,2 \\
\hline $\begin{array}{l}\text { Diseño y empleo estrategias de } \\
\text { enseñanza-aprendizaje interculturales. }\end{array}$ & 31 & 7,0 & 220 & 49,8 & 175 & 39,6 & 13 & 2,9 & 3 & 0,7 \\
\hline $\begin{array}{l}\text { Desde mi posición como educador } \\
\text { busco alternativas y recursos que } \\
\text { contribuyan a eliminar las barreras } \\
\text { sociales e institucionales que impiden el } \\
\text { acceso a oportunidades educativas de } \\
\text { algunos grupos culturales. }\end{array}$ & 18 & 4,1 & 179 & 40,5 & 234 & 52,9 & 10 & 2,3 & 1 & 0,2 \\
\hline $\begin{array}{l}\text { Imparto mis clases en la misma lengua } \\
\text { de mis alumnos. }\end{array}$ & 321 & 72,6 & 64 & 14,5 & 57 & 12,9 & 0 & 0,0 & 0 & 0,0 \\
\hline
\end{tabular}

Tan importante es el conocimiento de las normas que rigen la educación intercultural como las habilidades del docente puestas en función de la implementación de estrategias que permitan alcanzar el propósito de la educación intercultural; este debe poseer probadas capacidades para convertirse en facilitador de ambientes de aprendizajes interculturales basados en la igualdad de derechos y el intercambio de saberes y ser mediador en la construcción de una mejor sociedad inclusiva sin ningún tipo de discriminación en aras de alcanzar el Buen Vivir del ecuatoriano (Espinoza, 2020). Además, las averiguaciones demuestran que la educación intercultural bilingüe en los colegios de Machala está caracterizada por el analfabetismo intercultural existente en los docentes, pues no dominan la lengua originaria de sus alumnos, desarrollando la instrucción en lengua castellana; lo que es declarado por el $87,1 \%$ de los docentes encuestados, quienes consideran que el dominio de las lenguas originarias es muy bajo $(72,6 \%)$ o bajo $(14,5 \%)$ sin tener presente que la educación en la propia lengua del alumno va más allá de la ventaja que representa para el logro de un aprendizaje revelador, que requiere de significados para el reordenamiento de las estructuras cognitivas del aprendiz.

Cada cultura posee sus propios sentimientos, creencias, concepciones y códigos expresados a través de las prácticas comunicativas en torno a la estructura y el uso del lenguaje, lo que configura su ideología lingüística; por lo cual renunciar al aprendizaje en su lengua significa el quebrantamiento de sus derechos e identidad (Hinojosa y López, 2018; Hahl y Löfström, 2016). Al respecto Aman (2017) y Espinoza et al. (2019) consideran que las aspiraciones de lograr una educación intercultural bilingüe es un simple eufemismo, pues existe el analfabetismo intercultural por parte de muchos docentes, estos desconocen las lenguas y costumbres de los pueblos ancestrales; de igual forma, se cuenta con pocos educadores indígenas; por lo que en esencia se 
mantiene en la práctica la tradicional educación "occidental” bajo el nombre de educación intercultural bilingüe. En resumen, la información recogida en la tabla 4 revelan la tendencia de los docentes encuestados a percibir las acciones, habilidades y procedimientos empleados en función de la actividad pedagógica relacionada con la interculturalidad como de un nivel bajo; lo que apunta a la asimetría entre los indicadores de la dimensión cognitiva y la dimensión procedimental, marcando un distanciamiento entre la teoría y la práctica.

\section{Dimensión actitudinal}

En la tabla 5 se recogen de manera sintética los criterios de los docentes de la Educación Básica en las escuelas de la ciudad de Machala sobre sus actitudes y valores concernientes a la dimensión actitudinal de las competencias interculturales.

Tabla 5: Resultados de la dimensión actitudinal.

\begin{tabular}{|c|c|c|c|c|c|c|c|c|c|c|}
\hline \multirow{3}{*}{ Indicador } & \multicolumn{10}{|c|}{ Escala } \\
\hline & \multicolumn{2}{|c|}{ Muy Bajo } & \multicolumn{2}{|c|}{ Bajo } & \multicolumn{2}{|c|}{ Medio } & \multicolumn{2}{|c|}{ Alto } & \multicolumn{2}{|c|}{ Muy Alto } \\
\hline & Cant. & $\%$ & Cant. & $\%$ & Cant. & $\%$ & \begin{tabular}{|l} 
Cant. \\
\end{tabular} & $\%$ & Cant. & $\%$ \\
\hline $\begin{array}{l}\text { Percepción de sus sentimientos sobre los } \\
\text { diversos contextos socioculturales. }\end{array}$ & 0 & 0,0 & 11 & 2,5 & 339 & 76.7 & 86 & 19,5 & 6 & 1,4 \\
\hline $\begin{array}{l}\text { Percepción de los sentimientos de sus } \\
\text { alumnos sobre los contextos socioculturales } \\
\text { diferentes. }\end{array}$ & 2 & 0,5 & 7 & 1,6 & 321 & 72.6 & 79 & 17,9 & 33 & 7,5 \\
\hline $\begin{array}{l}\text { Identifico la cultura a la cual pertenezco y el } \\
\text { significado de pertenecer a ella, así como } \\
\text { las relaciones entre las personas de mi } \\
\text { grupo cultural con las personas de otros } \\
\text { grupos culturales, históricos, educativos, } \\
\text { sociales y políticos. }\end{array}$ & 0 & 0,0 & 0 & 0,0 & 236 & 53.4 & 189 & 42,8 & 17 & 3,8 \\
\hline $\begin{array}{l}\text { Respeto la cultura a la que pertenecen los } \\
\text { alumnos y el significado que representa } \\
\text { pertenecer a ella. }\end{array}$ & 0 & 0,0 & 0 & 0,0 & 246 & 55.7 & 160 & 36,2 & 36 & 8,1 \\
\hline $\begin{array}{l}\text { Identifico las actitudes, creencias y valores } \\
\text { que demuestran mi respeto y valoración } \\
\text { hacia las otras culturas, así como las que me } \\
\text { impiden respetar o valorarlas. }\end{array}$ & 0 & 0,0 & 0 & 0,0 & 245 & 55.4 & 188 & 42,5 & 9 & 2,0 \\
\hline $\begin{array}{l}\text { Soy capaz de corregir las actitudes y } \\
\text { creencias que no me permiten respetar o } \\
\text { valorar las diferencias de otros grupos } \\
\text { étnicos y culturales. }\end{array}$ & 10 & 2,3 & 101 & 22,9 & 186 & 42.1 & 143 & 32,4 & 2 & 0,5 \\
\hline $\begin{array}{l}\text { Tengo prejuicios sobre las personas que son } \\
\text { de otro grupo cultural. }\end{array}$ & 107 & 24,2 & 60 & 13,6 & 275 & 62.2 & 0 & 0,0 & 0 & 0,0 \\
\hline $\begin{array}{l}\text { Respeto las creencias de mis alumnos, } \\
\text { porque comprendo que éstas influyen tanto } \\
\text { en mi visión del mundo como en mi práctica } \\
\text { educativa. }\end{array}$ & 0 & 0,0 & 0 & 0,0 & 341 & 77.1 & 97 & 21,9 & 4 & 0,9 \\
\hline $\begin{array}{l}\text { Respeto la cosmovisión y conozco las } \\
\text { estructuras sociales de las comunidades } \\
\text { donde presto mis servicios de educador. }\end{array}$ & 0 & 0,0 & 0 & 0,0 & 102 & 23.1 & 328 & 74,2 & 12 & 2,7 \\
\hline $\begin{array}{l}\text { Promuevo en alumnos y otras figuras } \\
\text { educativas los valores del bilingüismo. }\end{array}$ & 33 & 7,5 & 134 & 30,3 & 275 & 62.2 & 0 & 0,0 & 0 & 0,0 \\
\hline $\begin{array}{l}\text { Utilizo mis valores, actitudes y creencias } \\
\text { culturales para apoyar el desarrollo } \\
\text { educativo de mis alumnos. }\end{array}$ & 0 & 0,0 & 0 & 0,0 & 156 & 35.3 & 275 & 62,2 & 11 & 2,5 \\
\hline $\begin{array}{l}\text { Me relaciono con colegas de contextos } \\
\text { culturales diferentes y puedo mantener un } \\
\text { diálogo sobre las diferencias y preferencias } \\
\text { culturales. }\end{array}$ & 0 & 0,0 & 0 & 0,0 & 232 & 52.5 & 195 & 44,1 & 15 & 3,4 \\
\hline $\begin{array}{l}\text { Me siento responsable de educar a mis } \\
\text { alumnos sobre sus derechos, metas, } \\
\text { expectativas y proveen una adecuada } \\
\text { orientación cultural. }\end{array}$ & 0 & 0,0 & 0 & 0,0 & 75 & 17.0 & 352 & 79,6 & 15 & 3,4 \\
\hline
\end{tabular}

El comportamiento de la dimensión actitudinal según las opiniones de los docentes encuestados indica la existencia de una adecuada concepción desde el punto de vista axiológico de la interculturalidad; así se evidencia en los indicadores, respeto de la propia cultura y a la de los demás, rubro valorado entre medio y alto por el $96,2 \%$ de los docentes encuestados; de igual forma se valoran el respeto a la cultura a la cual 
pertenecen los alumnos (94,0\%); identificación de las actitudes, creencias y valores hacia otras culturas (97,9\%); influencia de las culturas de los alumnos en la visión del mundo y las prácticas educativas (99,0\%); respeto a la cosmovisión y estructuras comunitarias del contexto donde se realiza la labor educativa (97,3\%); empleo de los valores, actitudes y creencias culturales para apoyar el desarrollo educativo de los alumnos $97,5 \%)$; relación con colegas de contextos culturales diferentes $(96,6 \%)$ y sentimientos de responsabilidad ante la educación de los alumnos sobre sus derechos, metas, expectativas y orientación cultural $(96,6 \%)$.

Por otro lado, la percepción de los propios sentimientos y la de los alumnos sobre los diversos contextos socioculturales tiene una tendencia a ser considerados de un nivel medio. Además, el $37,8 \%$ de los encuestados perciben como muy bajos $(24,2 \%)$ o bajos $(13,6 \%)$ sus prejuicios sobre las personas que son de otro grupo cultural, no obstante, aún existe un número considerable de docentes (62.2\%) que reconocen tener algún tipo de prejuicio y aproximadamente la cuarta parte $(25,2 \%)$ de los encuestados considera que la posibilidad de corregir esta actitud es baja o muy baja. También, el $37,8 \%$ opina que realiza una baja o muy baja promoción, entre los alumnos y otras figuras educativas, de los valores del bilingüismo, lo que se corresponde lógicamente con el nivel de analfabetismo intercultural que predomina. Esta información, demuestra que, aunque existe un adecuado marco legal y conocimientos desde el punto de vista epistemológico, aún persisten ideas, creencias, opiniones y sentimientos encontrados sobre la esencia de la educación intercultural, lo que se evidencia en la práctica escolar.

Resultados que se corresponden con los obtenidos por Ricardo et al. (2017), quienes observaron que la mayoría de los profesores poseen conocimientos y sensibilidad hacia la cultura propia, respetan la cosmovisión de la cultura de sus alumnos y conocen las estructuras sociales de sus comunidades, aunque reconocen sus limitaciones en las metodologías, habilidades, capacidades y competencias para asumir la educación intercultural. Si se trata de mejorar el proceso educativo, es menester pensar en el papel clave que desempeñan los docentes, por lo que hay que prestar especial atención a su formación y luego a su constante superación postgraduada, de forma tal que se dote a los educadores de competencias necesarias y suficientes para implementar en la clase el enfoque de la educación intercultural (Feiman, 2001; Jon, 2009; Zwerg y Martínez, 2016).

En resumen, el estudio brinda información sobre las características de las competencias interculturales de los docentes de la Enseñanza Básica de la ciudad de Machala, que integraron la muestra, quienes perciben tener conocimientos y sensibilidad hacia la cultura propia y la de sus alumnos, pero poseen insuficiencias y limitaciones en las metodologías, habilidades y capacidades para asumir la educación intercultural, observándose un desequilibrio entre los indicadores de las dimensiones cognitiva y actitudinal respecto a la dimensión procedimental.

\section{CONCLUSIONES}

En correspondencia a los resultados, su discusión y análisis, se concluye que las competencias interculturales de los docentes muestreados, se caracterizan atendiendo a sus dimensiones, de la siguiente manera: 1) Dimensión cognitiva, poseer un nivel entre medio y alto de conocimientos sobre el marco legal que sustenta la educación intercultural; se significan: el dominio de la LOEI, percibido entre estas categorías por el 95,3\% de los docentes; conocimiento de las normas constitucionales en materia de interculturalidad $(90,5 \%)$ y las políticas educativas implementadas en la escuela $(87,8 \%)$, aunque se expresa muy bajo $(47,7 \%)$ o bajo $(27,4 \%)$ el conocimiento de las metodologías para alcanzar los objetivos de la educación intercultural. 2) Dimensión procedimental estimada entre muy baja o baja, dada por el criterio de los docentes que evalúan entre estas categorías la capacidad para la aplicación de técnicas de enseñanza-aprendizaje para instruir desde entornos multiculturales y el empleo experiencias educativas que permiten enriquecer los conocimientos y desarrollar las habilidades interculturales $(58,4 \%)$; el diseño y empleo de estrategias de enseñanza-aprendizaje interculturales $(56,8 \%)$ y la integración de los conocimientos desde la diversidad cultural y cosmovisión de los alumnos $(51,4 \%)$; así como, por el desconocimiento de las lenguas originarias en impartir las clases $(87,1 \%)$. 3) Dimensión actitudinal, percibida entre media y alta, dado por la obediencia de la propia cultura y a las relaciones con las demás (96,2\%); respeto a la cultura de los alumnos (94\%); respeto a la cosmovisión y estructuras comunitarias del contexto donde se realiza la labor educativa (97,3\%); empleo de los valores, actitudes y creencias culturales para educar a los alumnos (97,5\%); relación con colegas de contextos culturales diferentes $(96,6 \%)$ y sentimientos de responsabilidad ante la educación de los derechos, metas, expectativas y orientación cultural (96,6\%). Estos datos develan asimetría entre los indicadores de la dimensión cognitiva y actitudinal con los de la dimensión procedimental, marcando un distanciamiento entre la teoría y la práctica.

\section{REFERENCIAS}

Aguado, M.T., La educación intercultural: concepto, paradigmas, realizaciones. En Lecturas de Pedagogía Diferencial, 1 ā Edición., 89-104, Dykinson, Madrid, España (1991). 
Aman, R., Colonial Differences in Intercultural Education: on interculturality in the Andes and the decolonization of intercultural dialogue. doi: https://doi.org/10.1086/690459, Comparative Education Review, 61(1), 103-S120. (2017).

Asamblea Nacional Constituyente de la República del Ecuador, Constitución de la República del Ecuador. (Modificaciones). Quito: CEP. (2011).

Asamblea Nacional de la República del Ecuador, Ley Orgánica de Educación Intercultural (Modificación). Quito. (2016).

Boni, A. y Calabuig, C., Education for global citizenship at Universities. doi: https://doi.org/10.1177/1028315315602926. Journal of Studies in International Education, 21(1), 22-38. (2017).

Boni, A., Lopez-Fogues, A. y Walker, M., Higher education and the post-2015 agenda: A contribution from the human development approach. doi: https://doi.org/10.1080/17449626.2016.1148757, Journal of Global Ethics, $12(1), 17-28$. (2016).

Colmenero, R.J. y Pegalajar, P.M., Cuestionario para futuros docentes de Educación Secundaria acerca de las percepciones sobre atención a la diversidad: construcción y validación del instrumento. doi: 10.15581/004. Estudios sobre Educación. 29, 165-189. (2015).

Consejo de Educación Superior, Resolución del Consejo de Educación Superior 51. Reglamento de Regimen Academico Consejo Educacion Superior. Registro Oficial Edición Especial. 854. (2017).

Dervin, F., Towards post-intercultural teacher education: analysing 'extreme' intercultural dialogue to reconstruct interculturality. doi: 10.1080/02619768.2014.902441, Euro J. Teach Edu., 38(1), 71-86 (2015).

Espinoza, E., Herrera, L. y Castellano, J. La dimensión intercultural en la formación docente en Ecuador. doi 10.25115/psye.v10i1.2253. Psychology, Society y Education, 11(3), 341-354. (2019).

Espinoza, E. Intercultural dimension of public educational policies in pedagogic formation. Machala Technical University. Ecuator. Espacios, 41 (18) Page 26 (2020).

Feiman-Nemser, S., From preparation to practice: designing a continuum to strengthen and sustain teaching, In Teachers College Record, 103(6), 1013-1055, (2001).

Garita, A.V., La orientación intercultural: Una perspectiva para favorecer la convivencia en contextos multiculturales. doi: http://doi.org/10.15359/ree.18-1.13. Revista Electrónica Educare, 18(1), 281-291. (2014).

Hahl, K. y Löfström, E., Conceptualizing interculturality in multicultural teacher education. doi: https://doi.org/10.1080/17447143.2015.1134544, Journal of Multicultural Discourses, 11(3), 300-314. (2016).

Hajisoteriou, C., Intercultural education set forward: operational strategies and procedures in Cypriot classrooms. doi: 10.1080/14675986.2012.686022, Intercultural Edu., 23(2), 133-146 (2012).

Hernández-Sampieri, R., Fernández-Collado, C. y Baptista-Lucio, P., Metodología de la Investigación (6ta edición). México: McGrauHill. (2014).

Herrera, C., Pérez, C. y Echeita, G., Teorías implícitas y prácticas de enseñanza que promueven la inclusión educativa en la universidad. Instrumentos y antecedentes para la reflexión y discusión. doi: 10.4067/S0718-50062016000500006, Formación Universitaria, 9(5), 49-64 (2016).

Hinojosa, E. F., y López, M. C., Interculturality and Teacher Education. A Study from Pre-service Teachers' Perspective. doi: http://dx.doi.org/10.14221/ajte.2018v43n3.5. Australian Journal of Teacher Education, 43(3), 5. (2018).

Jon, J. E., 'Interculturality'in higher education as student intercultural learning and development: a case study in South Korea. doi: https://doi.org/10.1080/14675980903371308, Intercultural Education, 20(5), 439-449. (2009).

Krainer, A., Aguirre, D., Guerra, M. y Meise, A., Educación superior intercultural y diálogo de saberes: el caso de la Amawtay Wasi. doi: https://doi.org/10.1016/j.resu.2017.11.002, RESU, Revista de la Educación Superior, 46 (184), 55-76 (2017).

Levy-Leboyer, Gestión de competencias. Barcelona: Ediciones 2000, (1996).

Moreno, S., Antropología abierta, antropología ambigua. Tendencias actuales de la antropología ecuatoriana. Multiculturalismo e Interculturalidad en América Latina, $1^{\text {a }}$ edición 68-96, Coord. A. Barabas, México: INAH. (2018).

Ricardo, B.C., Llinás, S.H. y Hernández, B.S., Competencias interculturales de profesores virtuales en universidades de la Costa Caribe Colombiana. Opción, 33(82), 263-279. (2017).

Rueda, G., Paz, L. y Avendaño, W., Análisis de la Educación Intercultural en Grupos de Estudiantes de la Universidad Francisco de Paula Santander, en Colombia, que Fueron Víctimas del Conflicto Armado, doi: http://dx.doi.org/10.4067/S0718-50062019000400095, Formación Universitaria, 12(4), 95-104 (2019)

Santoro, N., 'If I'm going to teach about the world, I need to know the world': developing Australian pre-service teachers' intercultural competence through international trips. doi: 10.1080/13613324.2013.832938, Race Ethnicity and Education, 17(3), 429-444. (2014).

Schweisfurth, M., y Gu, Q., Exploring the experiences of international students in UK higher education: possibilities and limits of interculturality in university life. doi: https://doi.org/10.1080/14675980903371332, Intercultural Education, 20(5), 463-473. (2009). 
Sleeter, C.E., Multiculturalism and education for citizenship in a context of neoliberalism. doi: 10.1080/14675986.2014.886357, Intercultural Edu., 25(2), 85-94 (2014).

Tarozzi, M., Building an "intercultural ethos" in teacher education. doi: 10.1080/14675986.2014.888804, Intercultural Edu., 25 (2), 128-142, (2014)

Uribe-Pérez, M. y Mosquera, C., La formación del profesorado desde el enfoque intercultural. Una aproximación a su estado actual. Episteme y Didaxis. No. Extraordinario, 1698-1704. (2016).

Zwerg, V. A. y Martínez D. J., Experiential Learning with Global Virtual Teams: Developing Intercultural and Virtual Competencies. doi: http://dx.doi.org/10.11144/Javeriana.m9-18. Magis, Revista Internacional de Investigación en Educación, 9(18), 129 -146. (2016). 